\title{
Fuzzy rank functions in the set of all binary systems
}

\author{
Hee Sik Kim', J. Neggers ${ }^{2}$ and Keum Sook So ${ }^{3^{*}}$
}

*Correspondence:

ksso@hallym.ac.kr

${ }^{3}$ Department

of Mathematics, Hallym

University, Chuncheon 24252,

Korea

Full list of author information

is available at the end of the

article

\section{Abstract}

In this paper, we introduce fuzzy rank functions for groupoids, and we investigate their roles in the semigroup of binary systems by using the notions of right parallelisms and $\rho$-shrinking groupoids.

Keywords: (Fuzzy) rank-subalgebra, (Fuzzy) rank-d-function, (Fuzzy) symmetric-rankfunction, Right parallel, $\rho$-Shrinking, Selective, $\operatorname{Bin}(X)$

Mathematics Subject Classification: Primary 08A72, 20N02

\section{Background}

The notion of a fuzzy subset of a set was introduced by Zadeh (1965). His seminal paper has opened up new insights and applications in a wide range of scientific fields. Rosenfeld (1971) used the notion of a fuzzy subset to set down corner stone papers in several areas of mathematics. Mordeson and Malik (1998) published a remarkable book, Fuzzy commutative algebra, presented a fuzzy ideal theory of commutative rings and applied the results to the solution of fuzzy intersection equations. The book included all the important work that has been done on $L$-subspaces of a vector space and on $L$-subfields of a field.

In the study of groupoids $(X, *)$ defined on set $X$, it has also proven useful to investigate the semigroups $(\operatorname{Bin}(X), \square)$ where $\operatorname{Bin}(X)$ is the set of all binary systems (groupoids) $(X, *)$ along with an associative product operation $(X, *) \square(X, \bullet)=(X, \square)$ such that $x \square y=(x * y) \bullet(y * x)$ for all $x, y \in X$. Thus, e.g., it becomes possible to recognize that the left-zero-semigroup $(X, *)$ with $x * y=x$ for all $x, y \in X$ acts as the identity of this semigroup [see Kim and Neggers (2008)]. Fayoumi (2011) introduced the notion of the center $Z \operatorname{Bin}(X)$ in the semigroup $\operatorname{Bin}(X)$ of all binary systems on a set $X$, and showed that a groupoid $(X, \bullet) \in Z \operatorname{Bin}(X)$ if and only if it is a locally-zero groupoid. Han et al. (2012) introduced the notion of hypergroupoids $(H \operatorname{Bin}(X), \square)$, and showed that $(H \operatorname{Bin}(X), \square)$ is a supersemigroup of the semigroup $(\operatorname{Bin}(X), \square)$ via the identification $x \longleftrightarrow\{x\}$. They proved that $\left(\operatorname{HBin}^{*}(X), \ominus,[\emptyset]\right)$ is a $B C K$-algebra.

In this paper, we introduce fuzzy rank functions for groupoids, and we investigate their roles in the semigroup of binary systems by using the notions of right parallelisms and $\rho$-shrinking groupoids. 


\section{Preliminaries}

Given a non-empty set $X$, we let $\operatorname{Bin}(X)$ denote the collection of all groupoids $(X, *)$, where $*: X \times X \rightarrow X$ is a map and where $*(x, y)$ is written in the usual product form. Given elements $(X, *)$ and $(X, \bullet)$ of $\operatorname{Bin}(X)$, define a product " $\square$ " on these groupoids as follows:

$$
(X, *) \square(X, \bullet)=(X, \square)
$$

where

$$
x \square y=(x * y) \bullet(y * x)
$$

for any $x, y \in X$. Using that notion, H. S. Kim and J. Neggers proved the following theorem.

Theorem 1 (Kim and Neggers 2008) (Bin $(X), \square)$ is a semigroup, i.e., the operation " $\square$ " as defined in general is associative. Furthermore, the left-zero-semigroup is the identity for this operation.

\section{Fuzzy rank functions for groupoids}

Given a groupoid $(X, *)$ in $\operatorname{Bin}(X)$, a map $\rho: X \rightarrow[0, \infty)($ or $\rho: X \rightarrow[0,1])$ is said to be:

(i) a (fuzzy) rank-subalgebra: $\rho(x * y) \geq \min \{\rho(x), \rho(y)\}$,

(ii) a (fuzzy) rank-co-subalgebra: $\rho(x * y) \leq \max \{\rho(x), \rho(y)\}$,

(iii) a (fuzzy) rank-d-function: $\rho(x * y)=\max \{\rho(x)-\rho(y), 0\}$,

(iv) a (fuzzy) symmetric-rank-function: $\rho(x * y)=\max \{\rho(x)-\rho(y), \rho(y)-\rho(x)\}$ for all $x, y \in X$.

Note that if $\rho: X \rightarrow[0, \infty)$ is a fuzzy subset of $X$, then it is a fuzzy rank-subalgebra as well. Thus these algebraic structures are special cases of the classes of fuzzy ranksubalgebras. Each of the types listed above serve as some idea of measure of size when the binary operation " $*$ " is considered as corresponding very roughly to a (group) sum (product), a left-zero-semigroup, a $d$-algebra ( $B C K$-algebra) or an absolute (value) difference. For the sake of being able to make comparisons in the behavior of interactions of rank-type and groupoid it seems a better idea to consider these simultaneously rather than study only isolated cases without considering common aspects as well as distinguishing ones.

Example 2 (a) Let $(X, *)$ be a left-zero-semigroup, i.e., $x * y=x$ for all $x, y \in X$. If $\rho: X \rightarrow[0, \infty)$ is any map, then $\rho(x * y)=\rho(x)$ for all $x, y \in X$. It follows that $\rho(x * y) \geq \min \{\rho(x), \rho(y)\}$ and $\rho(x * y) \leq \max \{\rho(x), \rho(y)\}$ for all $x, y \in X$, which shows that every function $\rho: X \rightarrow[0, \infty)$ is both a (fuzzy) rank-subalgebra and a (fuzzy) rankco-subalgebra.

(b) Let $(X, *)$ be a left-zero-semigroup and let $\rho: X \rightarrow[0, \infty)$ be a (fuzzy) rank- $d$-function. Then it is a zero function. In fact, if we assume that there exists an $x_{0} \in X$ such that $\rho\left(x_{0}\right)>0$, then $0<\rho\left(x_{0}\right)=\rho\left(x_{0} * y\right)=\max \left\{\rho\left(x_{0}\right)-\rho(y), 0\right\}$ for all $y \in X$. If we take $y:=x_{0}$, then it leads to a contradiction. 
(c) Let $(X, *)$ be a left-zero-semigroup and let $\rho: X \rightarrow[0, \infty)$ be a (fuzzy) symmetric-rank-function. Assume that there exists $x_{0} \in X$ such that $0<\rho\left(x_{0}\right)$. Since $\rho$ is a (fuzzy) symmetric-rank-function, we have $\rho(x)=\rho(x * y)=$ $\max \{\rho(x)-\rho(y), \rho(y)-\rho(x)\}$ for all $x, y \in X$. If we take $x:=x_{0}, y:=y_{0}$, then $0<\rho\left(x_{0}\right)=\rho\left(x_{0} * x_{0}\right)=\max \left\{\rho\left(x_{0}\right)-\rho\left(x_{0}\right), \rho\left(x_{0}\right)-\rho\left(x_{0}\right)\right\}=0, \quad$ a contradiction. This shows that $\rho$ is a zero function.

If $(X, *)$ is a right-zero-semigroup, i.e., $x * y=y$ for all $x, y \in X$, then any function $\rho: X \rightarrow[0, \infty)$ is both a (fuzzy) rank-subalgebra and a (fuzzy) rank-co-subalgebra of $(X, *)$, while if $\rho$ is either a (fuzzy) rank- $d$-function or a (fuzzy) symmetric-rank-function, then it is a zero function.

A groupoid $(X, *)$ is said to be selective (Neggers 1976; Neggers and Kim 1996) if $x * y \in\{x, y\}$ for all $x, y \in X$. For example, every left-(right-)zero-semigroup is selective. Given a selective groupoid $(X, *)$, we may construct a digraph via $x \rightarrow y \Leftrightarrow x * y=y$ for all $x, y \in X$. Hence selective groupoids are interpretable as digraphs on the (vertex) set $X$.

Proposition 3 Let $(X, *)$ be a selective groupoid and let $\rho: X \rightarrow[0, \infty)$ be a (fuzzy) rank-d-function of $X$. If $x \in X$ such that $\rho(x)>0$, then any vertex $y(\neq x)$ in $X$ with $x \nrightarrow y$ has $\rho(y)=0$.

Proof Let $y(\neq x)$ in $X$ with $x \nrightarrow y$. Since $(X, *)$ is selective and $x \nrightarrow y$, we have $x * y=x$. It follows from the fact that $\rho$ is a rank- $d$-function that $\rho(x)=\rho(x * y)=\max \{\rho(x)-\rho(y), 0\}$ and hence that $\rho(x)=\rho(x)-\rho(y)$, proving that $\rho(y)=0$.

Theorem 4 Let $(X, *)$ be a selective groupoid and let $\rho: X \rightarrow[0, \infty)$ be a (fuzzy) rank$d$-function of $X$. If $x \in X$ such that $\rho(x)>0$, then there exists at most one vertex $x \in X$ such that $\rho(x)>0$.

Proof Assume that there are two vertices $x$ and $y$ in $X$ such that $\rho(x)>0, \rho(y)>0$. It follows that $x \rightarrow y, y \rightarrow x$ by Proposition 3. Now $x \rightarrow y$ implies that $x * y=y$. Hence $0<\rho(y)=\rho(x * y)=\max \{\rho(x)-\rho(y), 0\}=\rho(x)-\rho(y)$. This shows that $2 \rho(y)=\rho(x)$. Similarly, $\quad y \rightarrow x \quad$ implies $\quad 2 \rho(x)=\rho(y)$. Thus $\quad$ we obtain $\rho(x)=2 \rho(y)=4 \rho(x)$, which implies $\rho(x)=0$, a contradiction.

Proposition 5 If $(X, *)$ is a selective groupoid, then every (fuzzy) rank-d-function of $(X, *)$ is a zero function.

Proof If $(X, *)$ is a selective groupoid, then $x * x=x$ for all $x \in X$. Since $\rho$ is a (fuzzy) rank- $d$-function, we obtain $\rho(x)=\rho(x * x)=\max \{\rho(x)-\rho(x), 0\}=0$, proving the proposition.

Given a map $\rho: X \rightarrow[0, \infty)$, we define

$$
\sum_{i}(\rho):=\{(X, *) \in \operatorname{Bin}(X) \mid \text { the condition }(i) \text { holds for }(X, *)\} \quad(i=1,2,3,4) .
$$

Proposition $6 \quad\left(\sum_{i}(\rho), \square\right)$ is a subsemigroup of $(\operatorname{Bin}(X), \square)$ where $i=1,2,3$. 
Proof Let $(X, *),(X, \bullet) \in \sum_{1}(\rho)$. Then $\rho(x * y) \geq \min \{\rho(x), \rho(y)\}$ and $\rho(x \bullet y) \geq$ $\min \{\rho(x), \rho(y)\}$ for all $x, y \in X$. If we let $(X, \square):=(X, *) \square(X, \bullet)$, then for any $x, y \in X$, we have $x \square y=(x * y) \bullet(y * x)$. It follows that

$$
\begin{aligned}
\rho(x \square y) & =\rho((x * y) \bullet(y * x)) \\
& \geq \min \{\rho(x * y), \rho(y * x)\} \\
& \geq \min \{\min \{\rho(x), \rho(y)\}, \min \{\rho(y), \rho(x)\}\} \\
& =\min \{\rho(x), \rho(y)\}
\end{aligned}
$$

This shows that $(X, *) \square(X, \bullet)=(X, \square) \in \sum_{1}(\rho)$. Hence $\left(\sum_{1}(\rho), \square\right)$ is a subsemigroup of $(\operatorname{Bin}(X), \square)$. Similarly, we may obtain that $\left(\sum_{i}(\rho), \square\right)$ is also a subsemigroup of $(\operatorname{Bin}(X), \square)$ where $i=2,3$.

Note that $\left(\sum_{4}(\rho), \square\right)$ need not be a subsemigroup of $(\operatorname{Bin}(X), \square)$. Let $(X, *),(X, \bullet) \in \sum_{1}(\rho)$ and let $(X, \square):=(X, *) \square(X, \bullet)$. If we take $x, y \in X$ such that $\rho(x)>\rho(y)$, then we have

$$
\begin{aligned}
\rho(x \square y)= & \rho((x * y) \bullet(y * x)) \\
= & \max \{\rho(x * y)-\rho(y * x), \rho(y * x)-\rho(y * x)\} \\
= & \max \{\max \{\rho(x)-\rho(y), \rho(y)-\rho(x)\} \\
& -\max \{\rho(y)-\rho(x), \rho(x)-\rho(y)\}, \\
& \max \{\rho(y)-\rho(x), \rho(x)-\rho(y)\} \\
& -\max \{\rho(x)-\rho(y), \rho(y)-\rho(x)\}\} \\
= & \min \{[\rho(x)-\rho(y)]-[\rho(x)-\rho(y)], \\
& {[\rho(x)-\rho(y)]-[\rho(x)-\rho(y)]\} } \\
= & 0<\rho(x)-\rho(y) \\
= & \max \{\rho(x)-\rho(y), \rho(y)-\rho(x)\},
\end{aligned}
$$

which shows that $\left(\sum_{4}(\rho), \square\right)$ is not a subsemigroup of $(\operatorname{Bin}(X), \square)$.

Let $\rho: X \rightarrow[0, \infty)$ be a map. A groupoid $(X, *)$ is said to have a $\rho$-chain $n$ if there exist $x_{1}, \ldots, x_{n} \in X$ such that $\rho\left(x_{1}\right)<\rho\left(x_{2}\right)<\cdots<\rho\left(x_{n}\right)$. We denote the $\rho$-chain by $\left\langle x_{1}, \ldots, x_{n}\right\rangle$. A groupoid $(X, *)$ is said to have the $\rho$-height $n$ if $\left\langle x_{1}, \ldots, x_{n}\right\rangle$ is the largest maximal $\rho$-chain in $(X, *)$.

Proposition 7 Let $\rho: X \rightarrow[0, \infty)$ be a map and let $\operatorname{Bin}(X)=\sum_{1}(\rho)$. Then the $\rho$ -height of $(X, *)$ is $\geq 2$ for any $(X, *) \in \operatorname{Bin}(X)$.

Proof Assume there exist $x, y, z \in X$ such that $\rho(x)<\rho(y)<\rho(z)$. Let $(X, *)$ be a groupoid such that $x=y * z$. Then $(X, *) \in \operatorname{Bin}(X)=\sum_{1}(\rho)$. It follows that $\rho(y * z)=\rho(x)<\rho(y)=\min \{\rho(y), \rho(z)\}$. This shows that $(X, *) \notin \sum_{1}(\rho)=\operatorname{Bin}(X)$, a contradiction.

Proposition 8 Let $\rho: X \rightarrow[0, \infty)$ be a map and let $\operatorname{Bin}(X)=\sum_{1}(\rho)$. If $\rho$ has two values $a, b \in[0, \infty)$ with $a<b$, then there exists uniquely an $\widehat{x} \in X$ such that $\rho(\widehat{x})=b$ and $\rho(y)=$ a for all $y \neq \widehat{x}$ in $X$.

Proof Let $\widehat{x} \in X$ such that $\rho(\widehat{x})=b>a$. If $y \in X$ such that $\widehat{x} \neq y$, then $\rho(y)=a$. In fact, if $\rho(y)=b$, then we may take a groupoid $(X, *)$ in $\operatorname{Bin}(X)$ such that $\rho(\widehat{x} * y)=a$, 
since $\operatorname{Bin}(X)=\sum_{1}(\rho)$. It follows that $a=\rho(\widehat{x} * y) \geq \min \{\rho(\widehat{x}), \rho(y)\}=b$, a contradiction. We claim that such an $\widehat{x}$ is unique. Assume that there are two elements $x^{\prime}, \widehat{x}$ in $X$ such that $\rho(\widehat{x})=b=\rho\left(x^{\prime}\right)$. A groupoid $(X, \bullet)$ satisfying $\rho\left(\widehat{x} \bullet x^{\prime}\right)=a$ may then be selected. It follows that $a=\rho\left(\widehat{x} \bullet x^{\prime}\right) \geq \min \left\{\rho(\widehat{x}), \rho\left(x^{\prime}\right)\right\}=b$, a contradiction.

Proposition 9 Let $(X, *) \in \operatorname{Bin}(X)$ and let $\rho: X \rightarrow[0, \infty)$ be a (fuzzy) rank-d-function of $X$. Then

(1) $\operatorname{Ker}(\rho) \neq \emptyset$,

(2) if $\rho(x) \leq \rho(y)$ then $x * y \in \operatorname{Ker}(\rho)$,

(3) $\operatorname{Ker}(\rho)$ is a right ideal of $(X, *)$.

Proof (1) Given $x \in X, \rho(x * x)=\max \{\rho(x)-\rho(x), 0\}=0$. It follows that $x * x \in \operatorname{Ker}(\rho)$. (2) If $\rho(x) \leq \rho(y)$ then $\rho(x * y)=\max \{\rho(x)-\rho(y), 0\}=0$ and hence $x * y \in \operatorname{Ker}(\rho)$. (3) Given $x \in \operatorname{Ker}(\rho), y \in X$, wehave $\rho(x * y)=\max \{\rho(x)-\rho(y), 0\}=\max \{0-\rho(y), 0\}=0$, which shows $x * y \in \operatorname{Ker}(\rho)$.

\section{Right parallelisms}

Let $\rho_{1}, \rho_{2}$ be mappings from $X$ to $[0, \infty)$. The map $\rho_{1}$ is said to be right parallel to $\rho_{2}$ if $\rho_{1}(a) \leq \rho_{1}(b)$ implies $\rho_{2}(a) \leq \rho_{2}(b)$, and we denote it by $\rho_{1} \| \rho_{2}$.

Proposition 10 If $\rho_{1}$ is a (fuzzy) rank-subalgebra of $(X, *)$ and $\rho_{1} \| \rho_{2}$, then $\rho_{2}$ is also a (fuzzy) rank-subalgebra of $(X, *)$.

Proof If $\rho_{1}$ is a rank-subalgebra of $(X, *)$, then $\rho_{1}(x * y) \geq \min \left\{\rho_{1}(x), \rho_{1}(y)\right\}$ for all $x, y \in X$. Without loss of generality, we let $\rho_{1}(x * y) \geq \rho_{1}(x)$. Since $\rho_{1} \| \rho_{2}$, we obtain $\rho_{2}(x * y) \geq \rho_{2}(x)$. It follows that $\rho_{2}(x * y) \geq \min \left\{\rho_{2}(x), \rho_{2}(y)\right\}$.

Given maps $\rho_{i}: X \rightarrow[0, \infty)$, we define

$$
\begin{aligned}
& \left(\rho_{1}+\rho_{2}\right)(x):=\rho_{1}(x)+\rho_{2}(x) \\
& \left(\rho_{1} \bullet \rho_{2}\right)(x):=\rho_{1}(x) \rho_{2}(x)
\end{aligned}
$$

for all $x \in X$.

Note that $\rho_{1}+\rho_{2}$ need not be a (fuzzy) rank-subalgebra of $(X, *)$ even though $\rho_{1}$ and $\rho_{2}$ are (fuzzy) rank-subalgebras of $(X, *)$. In fact,

$$
\begin{aligned}
\left(\rho_{1}+\rho_{2}\right)(x * y) & =\rho_{1}(x * y)+\rho_{2}(x * y) \\
& \geq \min \left\{\rho_{1}(x), \rho_{1}(y)\right\}+\min \left\{\rho_{2}(x), \rho_{2}(y)\right\}
\end{aligned}
$$

and

$$
\min \left\{\left(\rho_{1}+\rho_{2}\right)(x),\left(\rho_{1}+\rho_{2}\right)(y)\right\}=\min \left\{\rho_{1}(x)+\rho_{2}(x), \rho_{1}(y)+\rho_{2}(y)\right\}
$$

In the real numbers, it is not always true that $\min \{a, b\}+\min \{c, d\} \geq \min \{a+c, b+d\}$, which shows that $\rho_{1}+\rho_{2}$ need not be a (fuzzy) rank-subalgebra of $(X, *)$. 
Proposition 11 Let $\rho_{1}, \rho_{2}$ be mappings from $X$ to $[0, \infty)$. If $\rho_{1}$ is a constant for all $x \in X$ and if $\rho_{1} \| \rho_{2}$, then $\rho_{2}$ is also a constant function on $X$.

Proof Straightforward.

Note that any map $\rho_{1}$ is right parallel to $\rho_{2}$ if $\rho_{2}$ is a constant function. The right parallel' relation "II" is reflexive and transitive, but it is not an anti-symmetric, i.e., || is a quasi order, but not a partial order on $\{\rho \mid \rho: X \rightarrow[0, \infty):$ a map $\}$.

For example, we let $X:=[0, \infty)$ and let $\rho_{1}: X \rightarrow[0, \infty)$ be the identity map, and let $\rho_{2}: X \rightarrow[0, \infty)$ be a map defined by $\rho_{2}(x):=e^{x}$. Then $\rho_{1}(a) \leq \rho_{1}(b)$ if and only if $\rho_{2}(a) \leq \rho_{2}(b)$ for all $a, b \in X$, i.e., $\rho_{1}\left\|\rho_{2}, \rho_{2}\right\| \rho_{1}$, but $\rho_{1} \neq \rho_{2}$.

Proposition 12 If $\rho_{1}$ is a (fuzzy) rank-subalgebra of $(X, *)$ and $\rho_{1} \| \rho_{2}$, then $\rho_{1} \bullet \rho_{2}$ is also a (fuzzy) rank-subalgebra of $(X, *)$.

Proof If $\rho_{1}$ is a rank-subalgebra of $(X, *)$, then $\rho_{1}(x * y) \geq \min \left\{\rho_{1}(x), \rho_{1}(y)\right\}$ for all $x, y \in X$. If we assume $\rho_{1}(x * y) \geq \rho_{1}(x)$, then

$$
\begin{aligned}
\left(\rho_{1} \bullet \rho_{2}\right)(x * y) & =\rho_{1}(x * y) \rho_{2}(x * y) \\
& \geq \rho_{1}(x) \rho_{1}(y) \\
& =\left(\rho_{1} \bullet \rho_{2}\right)(x) \\
& \geq \min \left\{\left(\rho_{1} \bullet \rho_{2}\right)(x),\left(\rho_{1} \bullet \rho_{2}\right)(x)\right\}
\end{aligned}
$$

which proves the proposition.

Corollary 13 If $\rho_{1}$ is a (fuzzy) rank-co-subalgebra of $(X, *)$ and $\rho_{1} \| \rho_{2}$, then $\rho_{1} \bullet \rho_{2}$ and $\rho_{2}$ are also (fuzzy) rank-co-subalgebras of $(X, *)$.

Proof The proofs are similar to Propositions 10 and 12.

In the above note, we mentioned that $\rho_{1}+\rho_{2}$ need not be a rank-subalgebra of $(X, *)$. Using the notion of the right parallelism, we prove the following.

Proposition 14 If $\rho_{i}$ is a rank-subalgebra of $(X, *)(i=1,2)$ and if $\rho_{1} \| \rho_{2}$, then $\rho_{1}+\rho_{2}$ is also a rank-subalgebra of $(X, *)$.

Proof Since $\rho_{1}$ is a rank-subalgebra of $(X, *)$, we have $\rho_{1}(x * y) \geq \min \left\{\rho_{1}(x), \rho_{1}(y)\right\}$ for all $x, y \in X$. Without loss of generality, we let $\rho_{1}(x * y) \geq \rho_{1}(x)$. Since $\rho_{1} \| \rho_{2}$, we obtain $\rho_{2}(x * y) \geq \rho_{2}(x)$. It follows that $\rho_{1}(x * y)+\rho_{2}(x * y) \geq \rho_{1}(x)+\rho_{2}(x)$ and hence $\left(\rho_{1}+\rho_{2}\right)(x * y) \geq \min \left\{\left(\rho_{1}+\rho_{2}\right)(x),\left(\rho_{1}+\rho_{2}\right)(y)\right\}$, proving the proposition.

Corollary 15 If $\rho_{i}$ is a rank-co-subalgebra of $(X, *)(i=1,2)$ and if $\rho_{1} \| \rho_{2}$, then $\rho_{1}+\rho_{2}$ is also a rank-co-subalgebra of $(X, *)$.

Proof Similar to Proposition 14. 
Proposition 16 Let $X:=\mathbf{R}$ be the set of all real numbers. Let $\rho_{1}, \rho_{2}$ be mappings from $X$ to $[0, \infty)$ and let $\rho_{2}(x):=x$ for all $x \in X$. If $\rho_{1} \| \rho_{2}$, then $\rho_{1}$ is strictly increasing.

Proof Assume that there are $x, y \in X$ such that $x<y, \rho_{1}(x) \geq \rho_{1}(y)$. Since $\rho_{1} \| \rho_{2}$, we obtain $x=\rho_{2}(x) \geq \rho_{2}(y)=y$, a contradiction.

Theorem 17 If $\rho_{i}$ is a rank-d-function of $(X, *)(i=1,2)$ and if $\rho_{1} \| \rho_{2}$, then $\rho_{1}+\rho_{2}$ is also a rank-d-function of $(X, *)$.

Proof Given $x, y \in X$, we have four cases: (i) $\rho_{1}(x) \geq \rho_{1}(y), \rho_{2}(x) \geq \rho_{2}(y)$; (ii) $\rho_{1}(x) \geq \rho_{1}(y), \rho_{2}(x)<\rho_{2}(y)$; $\quad$ (iii) $\quad \rho_{1}(x)<\rho_{1}(y), \rho_{2}(x) \geq \rho_{2}(y)$; (iv) $\rho_{1}(x)<\rho_{1}(y)$, $\rho_{2}(x)<\rho_{2}(y)$. Since $\rho_{1} \| \rho_{2}$, the cases (ii) and (iii) are removed. For the case (i), we have $\rho_{1}(x * y)=\rho_{1}(x)-\rho_{1}(y)$ and $\rho_{2}(x * y)=\rho_{2}(x)-\rho_{2}(y)$. It follows that

$$
\begin{aligned}
\left(\rho_{1}+\rho_{2}\right)(x * y) & =\rho_{1}(x * y)+\rho_{2}(x * y) \\
& =\rho_{1}(x)-\rho_{1}(y)+\rho_{2}(x)-\rho_{2}(y) \\
& =\left(\rho_{1}+\rho_{2}\right)(x)-\left(\rho_{1}+\rho_{2}\right)(y) \\
& \leq \max \left\{\left(\rho_{1}+\rho_{2}\right)(x)-\left(\rho_{1}+\rho_{2}\right)(y), 0\right\},
\end{aligned}
$$

For the case (iv), we have $\rho_{1}(x * y)=0=\rho_{2}(x * y)$. It follows that $\left(\rho_{1}+\rho_{2}\right)(x * y)=\rho_{1}(x * y)+\rho_{2}(x * y)=0=\max \left\{\left(\rho_{1}+\rho_{2}\right)(x)-\left(\rho_{1}+\rho_{2}\right)(y), 0\right\}$, proving the theorem.

Note that if $\rho_{1}\left\|\rho_{2}, \rho_{2}\right\| \rho_{3}$, then $\rho_{1} \| \rho_{2}+\rho_{3}$, and if $\rho_{1}\left\|\rho_{2}, \rho_{1}\right\| \rho_{3}$, then $\rho_{1} \| \rho_{2}+\rho_{3}$.

\section{$\rho$-Shrinking groupoids}

A groupoid $(X, *)$ is said to be $\rho$-shrinking if $\rho: X \rightarrow[0, \infty)$ is a map satisfying the condition:

$$
\rho(x * y) \leq \min \{\rho(x), \rho(y)\}
$$

for all $x, y \in X$.

Example 18 Let $X:=[0, \infty)$ and let $x * y:=x+y$ for all $x, y \in X$. If we define $\rho(x):=x$ for all $x \in X$, then $\rho(x * y) \geq \max \{\rho(x), \rho(y)\}$. It follows that $\left(e^{-\rho}\right)(x * y)=e^{-x} e^{-y} \leq \min \left\{e^{-x}, e^{-y}\right\}=\min \left\{\left(e^{-\rho}\right)(x),\left(e^{-\rho}\right)(y)\right\}$, which shows that $(X, *)$ is $e^{-\rho}$-shrinking.

Theorem 19 If $(X, *)$ is both $\rho_{1}$-shrinking and $\rho_{2}$-shrinking and if $\rho_{1} \| \rho_{2}$, then $(X, *)$ is both $\left(\rho_{1}+\rho_{2}\right)$-shrinking and $\rho_{1} \bullet \rho_{2}$-shrinking.

Proof Since $(X, *)$ is $\rho_{1}$-shrinking, we have $\rho_{1}(x * y) \leq \min \left\{\rho_{1}(x), \rho_{1}(y)\right\}$ for all $x, y \in X$. The condition $\rho_{1} \| \rho_{2}$ implies that $\rho_{2}(x * y) \leq \min \left\{\rho_{2}(x), \rho_{2}(y)\right\}$. Hence

$$
\begin{aligned}
\left(\rho_{1}+\rho_{2}\right)(x * y) & =\rho_{1}(x * y)+\rho_{2}(x * y) \\
& \leq \min \left\{\rho_{1}(x), \rho_{1}(y)\right\}+\min \left\{\rho_{2}(x), \rho_{2}(y)\right\}
\end{aligned}
$$


Without loss of generality, we may assume $\rho_{1}(x) \leq \rho_{1}(y)$. Then $\rho_{2}(x) \leq \rho_{2}(y)$, since $\rho_{1} \| \rho_{2}$. It follows that $\left(\rho_{1}+\rho_{2}\right)(x * y) \leq \rho_{1}(x)+\rho_{2}(y)=\left(\rho_{1}+\rho_{2}\right)(x)$. Hence $(X, *)$ is $\left(\rho_{1}+\rho_{2}\right)$-shrinking. Similarly, if we assume $\rho_{1}(x) \leq \rho_{1}(y)$, then

$$
\begin{aligned}
\left(\rho_{1} \bullet \rho_{2}\right)(x * y) & =\rho_{1}(x * y) \rho_{2}(x * y) \\
& \leq \rho_{1}(x) \rho_{1}(x) \\
& =\left(\rho_{1} \bullet \rho_{2}\right)(x) \\
& =\min \left\{\left(\rho_{1} \bullet \rho_{2}\right)(x),\left(\rho_{1} \bullet \rho_{2}\right)(y)\right\},
\end{aligned}
$$

which shows that $(X, *)$ is $\rho_{1} \bullet \rho_{2}$-shrinking.

Proposition 20 If $(X, *)$ and $(X, \bullet)$ are $\rho$-shrinking and if $(X, \square):=(X, *) \square(X, \bullet)$, then $(X, \square)$ is also $\rho$-shrinking.

Proof If $(X, \square):=(X, *) \square(X, \bullet)$, then for all $x, y \in X$, we have

$$
\begin{aligned}
\rho(x \square y) & =\rho((x * y) \bullet(y * x)) \\
& \leq \min \{\rho(x * y), \rho(y * x)\} \\
& \leq \min \{\min \{\rho(x), \rho(y)\}, \min \{\rho(y), \rho(x)\} \\
& =\min \{\rho(x), \rho(y)\},
\end{aligned}
$$

showing that $(X, \square)$ is $\rho$-shrinking.

Proposition 20 shows that the collection of all $\rho$-shrinking groupoids forms a subsemigroup of $(\operatorname{Bin}(X), \square)$.

Given maps $\rho: X \rightarrow[0, \infty)$ and $\sigma: Y \rightarrow[0, \infty)$, we define a map $[\rho, \sigma]: X \times Y \rightarrow[0, \infty)$ by $[\rho, \sigma](x):=\rho(x)+\sigma(y)$ as a sort of "inner product" ranking. Given groupoids $(X, *)$ and $(Y, \bullet)$, we define a Cartesian product $(X \times Y, \nabla)$ where $(x, y) \nabla\left(x^{\prime}, y^{\prime}\right):=\left(x * x^{\prime}, y \bullet y^{\prime}\right)$ for all $(x, y),\left(x^{\prime}, y^{\prime}\right) \in X \times Y$.

Proposition 21 If $(X, *)$ is $\rho$-shrinking and $(Y, \bullet)$ is $\sigma$-shrinking, then $(X \times Y, \nabla)$ is $[\rho, \sigma]$ -shrinking.

Proof Since $(X, *)$ is $\rho$-shrinking and $(Y, \bullet)$ is $\sigma$-shrinking, we have $\rho(x * y) \leq \min \{\rho(x), \rho(y)\}$ and $\sigma(x \bullet y) \leq \min \{\sigma(x), \sigma(y)\}$ for all $x, y \in X$. It follows that

$$
\begin{aligned}
\rho(x * y)+\sigma(x \bullet y) & \leq \min \{\rho(x), \rho(y)\}+\min \{\sigma(x), \sigma(y)\} \\
& \leq \min \{\rho(x)+\sigma(x), \rho(y)+\sigma(y)\} \\
& =\min \{[\rho, \sigma](x),[\rho, \sigma](y)\},
\end{aligned}
$$

which proves the proposition.

\section{Conclusions}

Above, we introduced four (fuzzy) rank functions in the semigroup of all binary systems (i.e., groupoids), and we investigated their roles related to selective groupoids and the notion of $\operatorname{Bin}(X)$. Using the notion of "right parallelism", we showed that if $\rho_{i}$ is a (fuzzy) rank-subalgebra (resp., (fuzzy) rank- $d$-function) of $(X, *)(i=1,2)$ and if $\rho_{1} \| \rho_{2}$, then $\rho_{1}+\rho_{2}$ is also a (fuzzy) rank-subalgebra (resp., (fuzzy) rank- $d$-function) of $(X, *)$. 
By introducing the notion of $\rho$-shrinking to groupoids, we found that if $(X, *)$ is both $\rho_{1}$ -shrinking and $\rho_{2}$-shrinking and if $\rho_{1} \| \rho_{2}$, then it is both $\left(\rho_{1}+\rho_{2}\right)$-shrinking and $\rho_{1} \bullet \rho_{2}$ -shrinking. This research may provide hyper-fuzzy rank functions in the set of all binary systems naturally, and thus several well-developed theorems/propositions in the areas of soft fuzzy theory and intuitionistic fuzzy set theory can then possibly be applied in future research also.

Authors' contributions

All authors read and approved the final manuscript.

\section{Author details}

${ }^{1}$ Department of Mathematics, Hanyang University, Seoul 04763, Korea. ${ }^{2}$ Department of Mathematics, University of Alabama, Tuscaloosa, AL 35487-0350, USA. ${ }^{3}$ Department of Mathematics, Hallym University, Chuncheon 24252, Korea.

\section{Competing interests}

The authors declare that they have no competing interests.

Received: 19 February 2016 Accepted: 13 October 2016

Published online: 24 October 2016

\section{References}

Fayoumi HF (2011) Locally-zero groupoids and the center of Bin (X). Commun Korean Math Soc 26:163-168

Han JS, Kim HS, Neggers J (2012) The hypergroupoid semigroups as generalizations of the groupoid semigroups. J Appl Math 2012:717698

Kim HS, Neggers J (2008) The semigroups of binary systems and some perspectives. Bull Korean Math Soc 45:651-661

Mordeson JN, Malik DS (1998) Fuzzy commutative algebra. World Scientific, Singapore

Neggers J (1976) Partially ordered sets and groupoids. Kyungpook Math J 16:7-20

Neggers J, Kim HS (1996) Modular posets and semigroups. Semigroup Forum 53:57-62

Rosenfeld A (1971) Fuzzy groups. J Math Anal Appl 35:512-517

Zadeh LA (1965) Fuzzy sets. Inf Control 8:338-353

\section{Submit your manuscript to a SpringerOpen ${ }^{\circ}$ journal and benefit from:}

- Convenient online submission

- Rigorous peer review

Immediate publication on acceptance

- Open access: articles freely available online

- High visibility within the field

- Retaining the copyright to your article 